\title{
Abaque pour le calcul des caractéristiques de l'écoulement dans la section de gorge d'un siphon
}

\section{A graph for the calculation of flow characteristics in siphon throat sections}

\author{
PAR J. VALEMBOIS \\ LABORATOIRE NATIONAL D'HYDRAULIQUE (CHATOU)
}

L'abaque présenté permet de déterminer rapidement les caractéristiques d̀ donner à la section de gorge d'un siphon (hauteur, rayon de courbure du seuil), compte tenu du débit recherché et de la dépression que l'on admet.

\section{Introduction}

On admet en général, pour calculer l'écoulement dans la section de gorge d'un siphon, les hypothèses suivantes (voir fig. 1):

- Il n'y a pas de perte de charge entre la retenue et la section $\mathrm{M}_{0} \mathrm{M}_{1}$;

- L'écoulement y est à potentiel, les lignes de courant et les profils d'intrados et d'extrados en $M_{0}$ et $M_{1}$ étant des cercles de centre 0 .

Ces hypothèses conduisent à des calculs dont les résultats, que nous indiquons ci-dessous, sont confirmés par l'expérience avec une précision suffisante.

\section{Notations}

- $h$ est la cote du plan d'eau dans la retenue, à un endroit de faible vitesse,

- $R_{0}$ est le rayon de courbure en $M_{0}, R_{1}$ le rayon de courbure en $M_{1}$, et $L=R_{1}-R_{0}$ est la hauteur de la gorge du siphon,

- $V_{0}$ étant la vitesse en $M_{0}$, on posera

$$
\mathrm{H}_{0}=\mathrm{V}_{0}^{2} / 2 \mathrm{~g}
$$

Si l'on admet en $\mathrm{M}_{0}$ une dépression $\mathrm{H}_{\mathrm{D}}$, on aura

$$
\mathrm{H}_{0}=\mathrm{H}_{\mathrm{D}}+h
$$

This graph enables a rapid determination to be made of the siphon throat characteristics (i.e. sill height and radius of curvature) required for given discharges and depression

Posons :

et

$$
q_{0}=\mathrm{H}_{0} \sqrt{2 g \mathrm{H}_{0}}
$$

$$
\Delta \mathrm{H}=\frac{p_{1}}{\rho g}-\frac{p_{2}}{\rho g}
$$

$p_{0}$ et $p_{1}$ étant les pressions en $\mathrm{M}_{0}$ et $\mathrm{M}_{1}$.

\section{Résultats du calcul}

1. Si $q$ est le débit par unité de largeur, on peut l'écrire :

$$
\frac{q}{q_{0}}=\frac{\mathrm{R}_{0}}{\mathrm{H}_{0}} \log _{n}\left(1+\frac{\mathrm{L}}{\mathrm{R}_{0}}\right)
$$

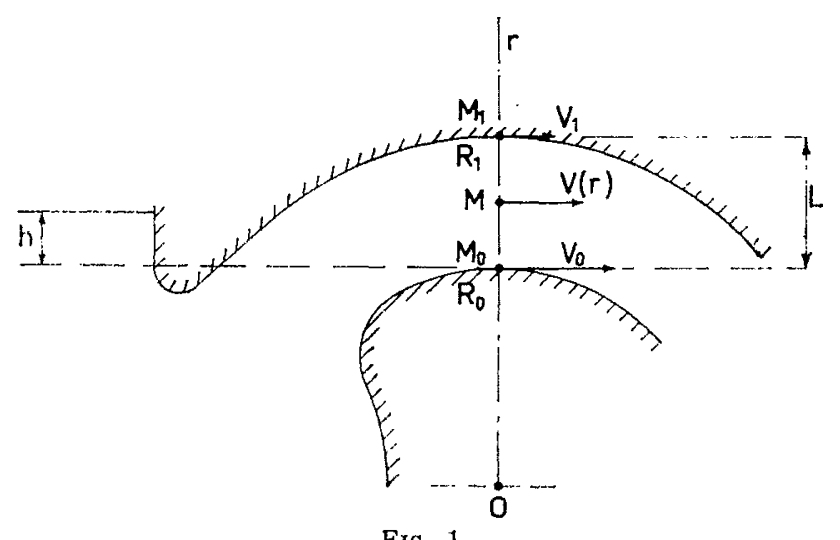

FIG. 1 


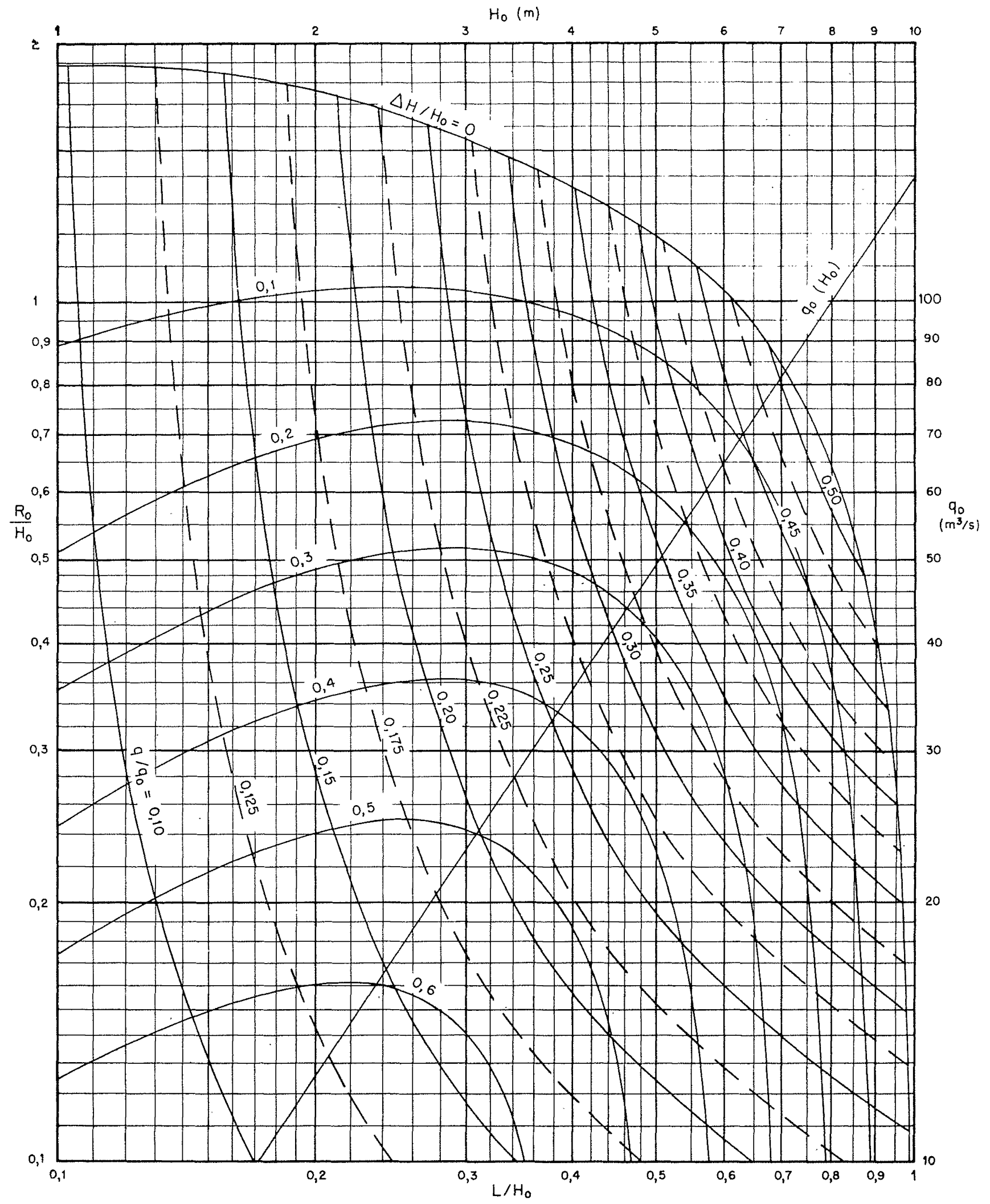

FIG. 2 
2. Ơn trouve aussi :

$$
\frac{\Delta H}{H_{0}}=1-\frac{1}{\left[1+\left(L / R_{0}\right)\right]^{2}}-\frac{L}{H_{0}}
$$

\section{Utilisation de l'abaque (fig. 2)}

L'abaque donne $q / q_{0}$ et $\Delta \mathrm{H} / \mathrm{H}_{0}$ en fonction de $\mathrm{L} / \mathrm{H}_{0}$ et $\mathrm{R}_{0} / \mathrm{H}_{0}$.

En général, on n'admettra pas que la pression en $M_{1}$ soit inférieure à la pression en $\mathbf{M}_{0}$. C'est pourquoi on a limité l'abaque aux valeurs positives de $\Delta \mathrm{H} / \mathrm{H}_{0}$.

On a porté aussi sur le même graphique $q_{0}$ en fonction de $\mathrm{H}_{0}$.

\section{Exemples}

1. Le débit maximal que l'on peut faire passer par $m$ de largeur s'obtient en admettant
$\Delta \mathrm{H}=0$,
$\mathrm{L} / \mathrm{H}_{0}=0,8$
et $\mathrm{R}_{0} / \mathrm{H}_{0}=0,65$ tes.

Le tableau ci-dessous donne ces valeurs limi-

\begin{tabular}{|r|l|l|l|}
\hline $\mathrm{H}_{0_{m}}$ & $\mathrm{~L}_{m}$ & $\mathrm{R}_{0_{m}}$ & $q_{\mathrm{m} / \mathrm{s}}$ \\
\cline { 1 - 2 } 2 & 1,6 & 1,3 & 6,5 \\
4 & 3,2 & 2,6 & 18,5 \\
6 & 4,8 & 3,9 & 34 \\
8 & 6,4 & 5,2 & 52 \\
10 & 8 & 6,5 & 72 \\
\hline
\end{tabular}

Il faut bien noter que $\mathrm{H}_{0}$ n'est pas la dépression admise, mais la somme de celle-ci et de la surélévation $h$ du plan d'eau par rapport au seuil.

2. Admettons une gorge de $2 \mathrm{~m}$ de haut et une dépression de $6 \mathrm{~m}$, la surélévation $h$ étant de $0,5 \mathrm{~m}$.

On a

et

$$
\mathrm{H}_{0}=6,5 \mathrm{~m}
$$

$$
\begin{aligned}
q_{0} & =75 \mathrm{~m}^{2} / \mathrm{s} \\
\mathrm{L} / \mathrm{H}_{0} & =0,308 \# 0,31
\end{aligned}
$$

Avec un rayon $R_{0}$ de $2 \mathrm{~m}$, on aura

$$
\mathrm{R}_{0} / \mathrm{H}_{0}=0,31
$$

et $q / q_{0} \# 0,215$, donc $q \# 16 \mathrm{~m}^{2} / \mathrm{s}$.

Si l'on peut admettre $\mathrm{R}_{n}=3,25 \mathrm{~m}$, on a

$$
\mathrm{R}_{0} / \mathrm{H}_{0}=0,5
$$

et $q / q_{0} \# 0,24$ donc $q \# 18 \mathrm{~m}^{2} / \mathrm{s}$. On ne gagne de cette façon que $2 \mathrm{~m}^{3} / \mathrm{s}$ par mètre de largeur.

Dans le premier cas $\left(R_{0}=2 \mathrm{~m}\right)$, on a

$$
\Delta \mathrm{H} / \mathrm{H}_{0} \# 0,44 \text {, }
$$

donc $\Delta \mathrm{H}=0,44 \times 6,5=2,85 \mathrm{~m}$. La dépression en $M_{1}$ serait donc $6-2,85=3,15 \mathrm{~m}$.

Dans le deuxième cas, $\Delta H / H_{0}=0,31$. Alors $\Delta \mathrm{H} \# 2 \mathrm{~m}$ et la dépression en $\mathrm{M}_{1}$ est

$$
6-2=4 \mathrm{~m} \text {. }
$$

3. Reprenons les dimensions du premier cas $\mathrm{du} \S 2\left(\mathrm{~L}=2 \mathrm{~m}, \mathrm{R}_{0}=2 \mathrm{~m}, h=0,5 \mathrm{~m}\right)$ en admettant une dépression de $8 \mathrm{~m}$.

Alors

$$
\begin{aligned}
& \mathrm{H}_{0}=8,5 \mathrm{~m} \quad q_{0}=110 \mathrm{~m}^{2} / \mathrm{s} \\
& \mathrm{L} / \mathrm{H}_{0}=\mathrm{R}_{0} / \mathrm{H}_{0}=2 / 8,5=0,235
\end{aligned}
$$

d'où

$$
q / q_{0}=0,163 \quad \text { et } q \# 18 \mathrm{~m}^{2} / \mathrm{s}
$$

On a relativement peu d'intérêt à augmenter la dépression.

\section{Remarque}

En général, on se donnera $\mathrm{H}_{0}$, défini par la dépression admise, et $\mathrm{L}$ (pour des raisons constructives). On voit alors que l'on a intérêt à adopter la plus grande valeur admissible pour $R_{0}\left(\tan t\right.$ que $\Delta H / H_{0}$ reste positif).

Le débit maximal serait évidemment obtenu pour un rayon de courbure infini. Ce serait

$$
a_{m}=\mathrm{LV}_{0}=\mathrm{L} \sqrt{2 g \mathrm{H}_{0}}
$$

mais dans ce cas, la pression en $M_{1}$ serait inférieure à la pression en $\mathrm{M}_{0}$ (on aurait une répartition hydrostatique, avec $\Delta \mathrm{H}=-\mathrm{L}$ ).

On peut écrire $q$ de la façon suivante :

$$
q=q_{m} \frac{\mathrm{R}_{0}}{\mathrm{~L}} \log _{n}\left(1+\frac{\mathrm{L}}{\mathrm{R}_{0}}\right)
$$

Le tableau ci-dessous donne $q / q_{m}$ en fonction de $R_{f} / L$. Pour l'utiliser, il faudra évidemment se limiter à la valeur de $R_{0}$ correspondant à $\Delta H=0$. On trouvera cette valeur en utilisant l'abaque de la figure 2.

\begin{tabular}{|c|c|}
\hline $\mathrm{R}_{0} / \mathrm{L}$ & $q / q_{m}$ \\
\hline 0,1 & 0,24 \\
0,2 & 0,36 \\
0,3 & 0,44 \\
0,4 & 0,50 \\
0,5 & 0,55 \\
0,6 & 0,59 \\
0,7 & 0,62 \\
0,8 & 0,65 \\
0,9 & 0,67 \\
1,0 & 0,69 \\
1,5 & 0,77 \\
2 & 0,81 \\
3 & 0,86 \\
4 & 0,89 \\
5 & 0,91 \\
\hline
\end{tabular}

